\title{
Improving SHA-2 Hardware Implementations
}

\author{
Ricardo Chaves ${ }^{1,2}$, Georgi Kuzmanov ${ }^{2}$, \\ Leonel Sousa ${ }^{1}$, and Stamatis Vassiliadis ${ }^{2}$ \\ ${ }^{1}$ Instituto Superior Técnico/INESC-ID. Rua Alves Redol 9, \\ 1000-029 Lisbon, Portugal \\ http://sips.inesc-id.pt/ \\ ${ }^{2}$ Computer Engineering Lab, TUDelft. Postbus 5031, 2600 GA Delft, \\ The Netherlands \\ http://ce.et.tudelft.nl/
}

\begin{abstract}
This paper proposes a set of new techniques to improve the implementation of the SHA-2 hashing algorithm. These techniques consist mostly in operation rescheduling and hardware reutilization, allowing a significant reduction of the critical path while the required area also decreases. Both SHA 256 and SHA512 hash functions have been implemented and tested in the VIRTEX II Pro prototyping technology. Experimental results suggest improvements to related SHA256 art above $50 \%$ when compared with commercial cores and $100 \%$ to academia art, and above $70 \%$ for the SHA512 hash function. The resulting cores are capable of achieving the same throughput as the fastest unrolled architectures with $25 \%$ less area occupation than the smallest proposed architectures. The proposed cores achieve a throughput of $1.4 \mathrm{Gbit} / \mathrm{s}$ and $1.8 \mathrm{Gbit} / \mathrm{s}$ with a slice requirement of 755 and 1667 for SHA256 and SHA512 respectively, on a XC2VP30-7 FPGA.
\end{abstract}

Keywords: Cryptography, Hash functions, SHA-2 (256, 512), FPGA.

\section{Introduction}

Cryptography is becoming an essential part of most electronic equipments that require data storing or manipulation. However, the algorithms used to enforce this security are too demanding to be implemented in software for the current required processing speeds. To achieve the require processing capability hardware components have to be used. These hardware cores are usually implemented either in dedicated ASIC cores [1-3] or in reconfigurable devices [4-7]. In this paper we propose a new hardware implementation of the SHA-2 algorithm, used in authentication systems and in the validity check of data. Several techniques have been proposed to improve the implementation of the SHA-2 algorithm. The most relevant are:

- the usage of parallel counters or well balanced Carry save Adders (CSA), in order to improve the partial additions. In technologies, like reconfigurable devices that have dedicated data paths for improving addition, this technique is not always beneficial; 
- unrolling techniques that optimize the data dependency. This technique allows for an improvement in the throughput, however, it usually significantly increases the required circuit area $[2,8,6]$;

- delay balancing and the usage of improved addition units, since in this algorithm this is the critical operation;

- the usage of embedded memories to store the required constant values $\left(K_{t}\right)$;

- use of pipelining techniques, to achieve higher working frequencies. Due to highly dependent data computation the resulting throughput is usually not improved and more complex control logic is required $[2,9]$.

However, the performance of the SHA-2 algorithm can be further improved with other techniques. To achieve this goal, this paper proposes operation rescheduling, that allows for an efficient use of a pipelined structure without an increase in area, and hardware reutilization techniques that allow for resource saving.

Both implementations of the SHA256 and SHA512 hash functions suggest:

- throughput per Slice efficiency metric improvement of 53\% compared to commercial SHA256 cores, and more than $100 \%$ to current SHA256 academia art, and $77 \%$ for SHA512 implementations;

- a throughput of $1.4 \mathrm{Gbit} / \mathrm{s}$ for SHA256 and $1.8 \mathrm{Gbit} / \mathrm{s}$ for SHA512, with 755 and 1667 slices, on a XC2VP30-7 FPGA, respectively;

- 150 times speedup with respect to the software implementation.

The paper is organized as follows, Section 2 presents the SHA-2 algorithms. Section 3 describes the proposed design. The characteristics of FPGA implementations are presented in section 4 . Section 5 presents the obtained results and compares them to related art. Section 6 , concludes the paper with some final remarks.

\section{SHA-2 Hash Algorithm}

In 1993 the Secure Hash Standard (SHA) was first published by the NIST. In 1995 this algorithm was reviewed in order to eliminate some of the initial weakness, and in 2001 new Hashing algorithms were proposed. This new family of hashing algorithms known as SHA-2, use larger digest messages, making them more resistent to possible attacks and allowing them to be used with larger blocks of data, up to $2^{128}$ bits, e.g. in the case of SHA512. The SHA-2 hashing algorithm is the same for the SHA256, SHA224, SHA384, and SHA512 hashing functions, differing only in the size of the operands, the initialization vectors, and the size of the final digest message.

The following describes the SHA-2 algorithm applied to the SHA256 hash function, followed by the description of the SHA512 hash function, which differs mostly in the size of the operands, using 64-bit words instead of 32-bit. Note that SHA224 and SHA384 are computed as SHA256 and SHA512, respectively, with the final hash value truncated to the corresponding size, the Initialization Vector also differs. 


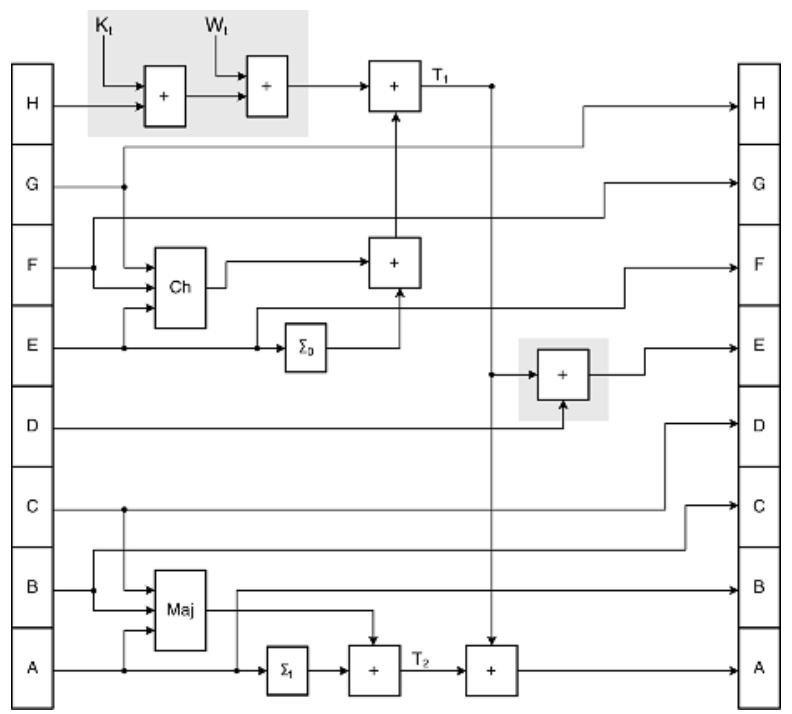

Fig. 1. SHA-2 round calculation

SHA256 Hash Function: The SHA256 Hash function produces a final digest message of 256 bits, that is dependent of the input message, composed by multiple blocks of 512 bits each. This input block is expanded and fed to the 64 cycles of the SHA256 function in words of 32 bits each ( denoted by $W_{t}$ ). In each cycle or round of the SHA-2 algorithm the introduced data is mixed with the current state. This data scrambling is preformed by additions and logical operations, such as bitwise logical operations and bitwise rotations. The computational structure of each round of this algorithm is depicted in Figure 1. The several functions presented in this figure are described in Appendix I. The value $W_{t}$ is the 32-bit data word, for the $t$ round, and the $K_{t}$ value represents the 32 -bit constant that also depends on the round.

The 32-bit values of the A to $\mathrm{H}$ variables are updated in each round and the new values are used in the following round. The initial values of these variables is given by the 256-bit constant value specified in [10], this value is only set for the first data block. The consecutive data blocks use the intermediate hash value, computed for the previous data block. Each 512 data block is processed for 64 rounds, after which the values of the variables $\mathrm{A}$ to $\mathrm{H}$ are added to the previous digest message, in order to obtain partial digest message. To better illustrate this algorithm a pseudo code representation is depicted in Figure 2. The final Digest Message $(D M)$ for a given data stream, is given by the result of the last data block.

In some higher level applications like the efficient implementation of the keyedHash Message Authentication Code (HMAC) [11] or when a message is fragmented, the initial hash value $(I V)$ may differ from the constant specified in [10]. In these cases, the variables $\mathrm{A}$ to $\mathrm{H}$ are initialized by a variable Initialization Vector $(I V)$. 


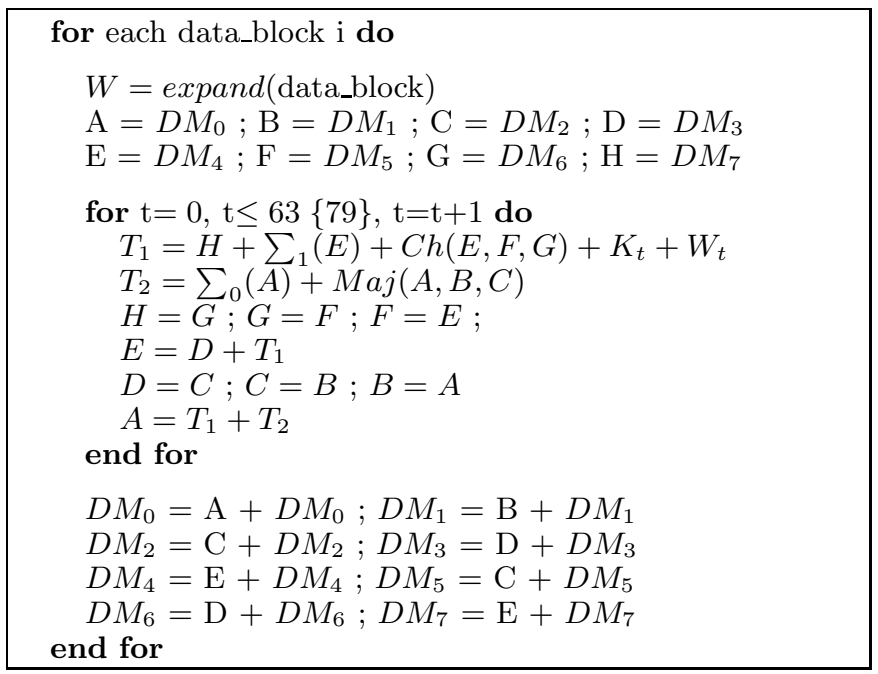

Fig. 2. Pseudo Code for SHA-2 algorithm

SHA512 Hash function: The SHA512 hash function computation is identical to that of the SHA256 hash function, differing in the size of the operands, that are of 64 bits and not 32 bits as for the SHA256, the size of the Digest Message, that has twice the size being composed by 512 bits, and in the $\Sigma$ functions described in Appendix I. This Appendix also describes the functions $\sigma$ used in the message schedule. The value $W_{t}$ and $K_{t}$ are of 64 bits and the each data block is composed by 16 64-bit words, having in total 1024 bits.

Message schedule: In the SHA-2 algorithm the computation described in Figure 1 is performed for 64 rounds for the SHA256 (80 rounds for the SHA512), in each round a 32-bit word (or 64-bit for SHA512) obtained from the intermidiate hash value is used. However each data block only has 16 32-bits words for SHA256 or 16 64-bit words for SHA512, resulting in the need to expand the initial data block to obtain the remaining words. This expansion is performed by the computation described in (1), where $M_{t}^{(i)}$ denotes the first 16 words of the i-th data block.

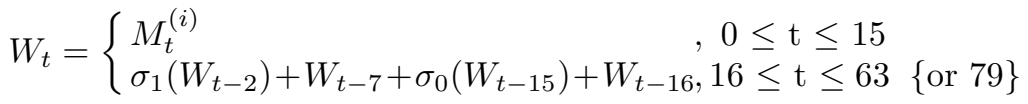

Message padding: In order to assure that the input message in a multiple of 512 bits, as required by the SHA256 hash function, or 1024 for the SHA512 hash function, it is necessary to pad the original message. This message padding also comprises the inclusion of the original message dimension to the padded message. This operation can be efficiently implemented in software with a minimal cost. 


\section{Proposed Design}

In the SHA-2 algorithm, the operations that have to be performed are simple, however the data dependency of this algorithm does not allow for much parallelization. Each round of the algorithm can only be computed after the values $A$ to $H$ of the previous round have been calculated (see figure 2), imposing a sequentiality to the computation. It should be noticed that in each round the computation is only required to calculate the values of $A$ and $E$, since the remaining values are obtained directly from the values of the previous round, as depicted in the pseudo code of Figure 2.

In this paper, we propose a new operation rescheduling technique, a new form to initialize the algorithm, and a more efficient hardware reutilization scheme.

Operation rescheduling: In our proposal, we identified the part of the computation of a given round $t$ that can be computed ahead in the previous round $t-1$. Only the values that do not depend on the values computed in the previous round can be computed ahead. Unlike the rescheduling technique proposed in [12] for the SHA1 algorithm, where the inter round data dependency is low, in the SHA-2 algorithm the data dependency is more complex, as depicted in Figure 1. While the variables $B, C, D, F, G$, and $H$ are obtained directly from the values of the round, not requiring any computation, the values of $A$ and $E$ require computation and depend on all the values. In other words, the values $A$ and $E$ for round $t$ can not be computed until the values for the same variables have been computed in the previous round have, as shown in (2).

$$
\begin{aligned}
& E_{t+1}=D_{t}+\Sigma_{1}\left(E_{t}\right)+C h\left(E_{t}, F_{t}, G_{t}\right)+H_{t}+K_{t}+W_{t} \\
& A_{t+1}=\Sigma_{0}\left(A_{t}\right)+\operatorname{Maj}\left(B_{t}, C_{t}, D_{t}\right)+\Sigma_{1}\left(E_{t}\right)+C h\left(E_{t}, F_{t}, G_{t}\right)+H_{t}+K_{t}+W_{t}
\end{aligned}
$$

Taking into account that the value $H_{t+1}$ is given directly by $G_{t}$ which in its turn is given by $F_{t-1}$, the precalculation of $H$ can thus be given by $H_{t+1}=F_{t-1}$. Since the value of $K_{t}$ and $W_{t}$ can be precalculated and are simply used in each round, (2) can be rewritten as:

$$
\begin{aligned}
\delta_{t} & =H_{t}+K_{t}+W_{t}=G_{t-1}+K_{t}+W_{t} ; \\
E_{t+1} & =D_{t}+\Sigma_{1}\left(E_{t}\right)+C h\left(E_{t}, F_{t}, G_{t}\right)+\delta_{t} ; \\
A_{t+1} & =\Sigma_{0}\left(A_{t}\right)+\operatorname{Maj}\left(B_{t}, C_{t}, D_{t}\right)+\Sigma_{1}\left(E_{t}\right)+C h\left(E_{t}, F_{t}, G_{t}\right)+\delta_{t},
\end{aligned}
$$

where the value $\delta_{t}$ is calculated in the previous round. The value $\delta_{t+1}$ can be the result of a full addition or the Carry and the Save vectors from a Carry Save Addition. With this computational separation the calculation of the SHA2 algorithm can be divided into two parts, allowing the calculation of $\delta$ to be rescheduled to the previous clock cycle, depicted by the grey area in Figure 3 . Thus the critical path of the resulting hardware implementation can be reduced. Since the computation is now divided by a pipeline stage, the calculation of the SHA-2 requires an additional clock cycle, to perform all the rounds. In the case of the SHA256 hash function 65 clock cycles are necessary to calculate the 64 rounds. As specified in the SHA-2 algorithm and depicted in Figure 2, after all 
rounds have been computed, the internal variables ( $\mathrm{A}$ to $\mathrm{H}$ ) have to be added to the previous Digest Message.

Hash value addition and initialization: As mentioned after the computation of a given data block, the internal variables have to be added to the intermediate hash value. If this addition were to be implemented in a straightforward manner, 8 adders would be required, one for each internal variable, of 32 or 64 bits depending if SHA256 or SHA512 is being implemented. However, some hardware reuse can be achieved. By analyzing the data dependency and the fact that most of the internal variables do not require any computation, since their value is given directly by the previous values of the other variables, taking into account that:

$$
\begin{aligned}
& H_{t}=G_{t-1}=F_{t-2}=E_{t-3} ; \\
& D_{t}=C_{t-1}=B_{t-2}=A_{t-3},
\end{aligned}
$$

the computation of the Digest Message for the data block $i$ can be calculated from the internal variables $A$ and $E$, as:

$$
\begin{aligned}
& D M 7_{i}=E_{t-3}+D M 7_{i-1} \quad ; \quad D M 3_{i}=A_{t-3}+D M 3_{i-1} ; \\
& D M 6_{i}=E_{t-2}+D M 6_{i-1} \quad ; \quad D M 2_{i}=A_{t-2}+D M 2_{i-1} ; \\
& D M 5_{i}=E_{t-1}+D M 5_{i-1} \quad ; \quad D M 1_{i}=A_{t-1}+D M 1_{i-1} .
\end{aligned}
$$

Thus the calculation can be performed by only 2 addition units, as:

$$
\begin{array}{clrl}
D M(j+4)_{i} & =E_{t-3+j}+D M(j+4)_{i-1} & & ; 1 \leq \mathrm{j} \leq 3 \\
D M(j)_{i} & =A_{t-3+j}+D M(j)_{i-1} & ; 1 \leq \mathrm{j} \leq 3 .
\end{array}
$$

The selection of the corresponding part of the Digest Message (DMj), could be performed by a multiplexer. However, taking into account the sequentiality in which the values of DMj are used, a shifting buffer can be used, as depicted in the right most part of Figure 3 . Since the values $A_{t}$ and $E_{t}$ require computation and the final value is only calculated in the last clock cycle, the calculation of the values $D M 0_{i}$ and $D M 4_{i}$ is performed in a different manner. Instead of using one full adder, after the calculation of the final value of $A$ and $E$, the Digest Message (DM) is added during the calculation of their final values, by a Carry Save Adder (CSA). Since the value of the previous Digest Message is known, the value can be added during the first stage of the pipeline, not being on the critical path, located in the second stage of the pipeline, where the full adders are used. In the last round the value of $A$ and $E$ is not calculated, being directly calculated the value of the Digest Message. During the normal round calculation only the values $A_{t}$ and $E_{t}$ can be computed, in these cases the input of the used CSA is put to zero, as depicted in Figure 3.

After each data block has been computed, the internal values $A$ to $H$ have to be re-initialized with the newly calculated Digest Message. This is performed by a multiplexer that selects either the new value of the variable of the Digest Message, as depicted in the left most side of Figure 3. Once more the values $A$ and $E$ are the exception. Since the final value computed for these two variables 

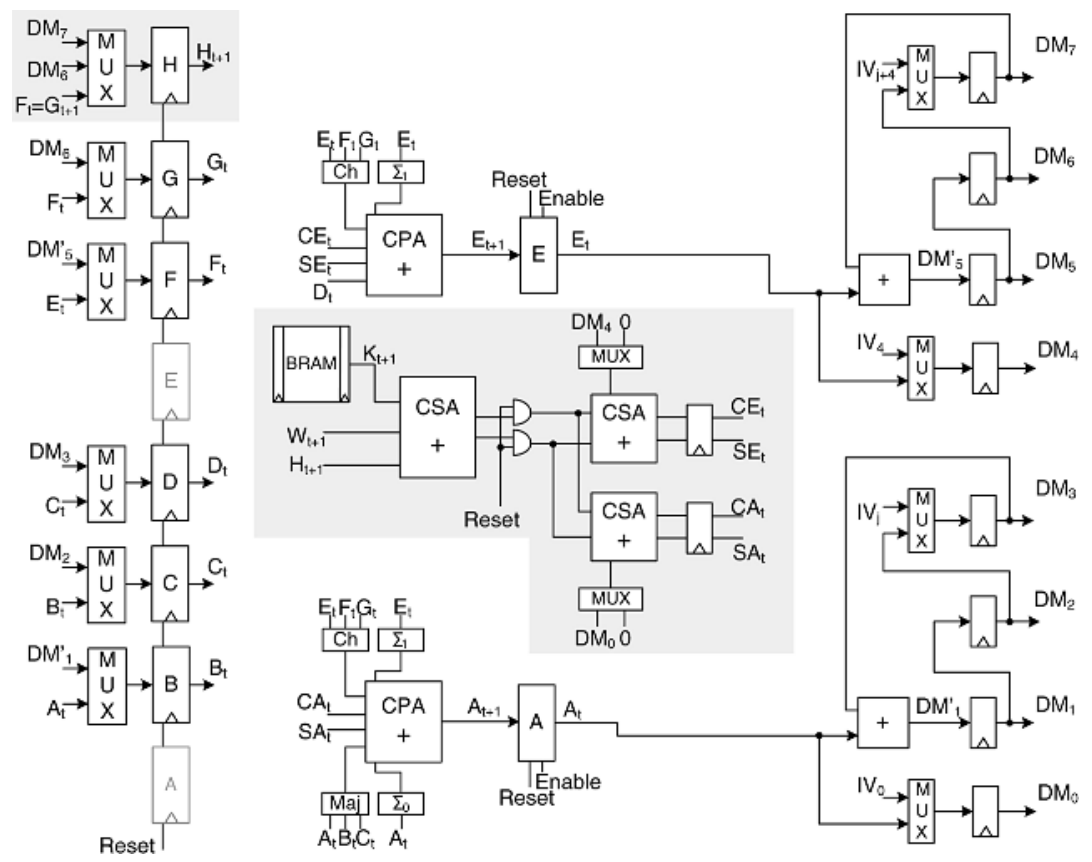

Fig. 3. SHA-2 round architecture

is already the Digest Message, the values are already loaded in the registers. An enable signal is used in the $A$ and $E$ registers, in order to maintain these values during the re-initialization of the other values.

In the first round the values of $A$ to $H$ also have to be initialized. All variables, except $A$ and $E$, are simply loaded with the values in the $D M$ registers, depicted in the leftmost part of Figure 3. For the $A$ and $E$ variables the value is fed through the round logic. In this case the, all the variables are set to zero (Reset) except the $D M_{0}$ and $D M_{4}$ inputs. Thus the resulting value for the $A$ and $E$ registers will be the initialization values of the $D M$ registers.

In the standard for the SHA-2 algorithm the initial value of the Digest Message (loaded to the $A$ to $H$ variables) is a constant value, that can be loaded by using set/reset signals in the registers. If the SHA-2 algorithm is to be used in a wider set of applications and in the computation of fragmented messages, the initial Digest Message is no longer a constant value. In these cases the initial value is given by the $I V$ that has to be loaded. This loading can be performed by multiplexers at the input of the Digest Message registers. In order to optimized the architecture the calculation structure for the Digest Message can be used to load the $I V$, not being directly loaded into all the registers. The value of the $A 1$ and $E 1$ registers is set to zero during this loading, thus the existing structure acts as a circular buffer, where the value is only loaded into one of the registers, and shifted to the others. 
This circular buffer can also be used to more efficiently read the final Digest Message, in a structure with an interface with smaller output ports, since the values are simply shifted and less multiplexes are required.

\section{SHA-2 FPGA Implementation}

In order to evaluate the proposed design, the resulting SHA256 and SHA512 hash functions cores have been implemented in a Xilinx VIRTEX II Pro (XC2VP307) FPGA using the Xilinx ISE (6.3) and SimplifyPro (8.4) tools. All the values presented for our cores were obtained after Place and Route. A Custom Computing Unit (CCU) using these SHA-2 cores, has also been designed for the Molen polymorphic computational model [13], in order to fully test the cores.

In order to fully exploit the capabilities of the reconfigurable device, some design adaptation can be made. The main one lays in the use of fast carry chains for Carry Propagate Adders (CPA) instead of CSA in both the first and in the second pipeline stage, since they are able to achieve the same performance in FPGA, with less area resources. For ASIC technologies, the structure depicted in Figure 3 is more suitable. When implementing the SHA256 hash function, a single BRAM can be used, since the 64 32-bits fit in a single 32-bit port embedded memory. However, in the SHA512 hash function the operands have 64 bits, including the constant $K_{t}$. Since the existing BRAMs do not have 64-bit ports, more than one would be required. However, they have a dual output ports of 32 bits each. Thus the 80 64-bit constants can be mapped as two 32-bit words: one port addresses the low part of the memory, with the lower 32 bits of the constant and the other the high part of the memory with the higher 32 bits of the same constant. With this, only one BRAM is used to generate the 64 bit constant.

For the message schedule in the FPGA technology considered, CPA are also used instead of CSA. The structure of the data expansion component is represented in Figure 4.

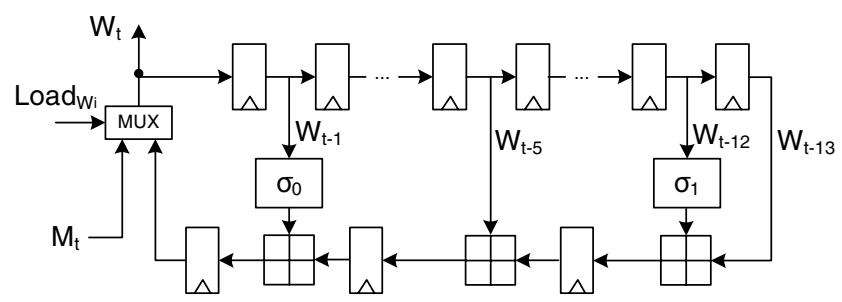

Fig. 4. SHA-2 data expansion module

These cores have also been integrated as a CCU for the MOLEN processor [13]. The MOLEN computational paradigm enables the SHA-2 core to be embedded in a reconfigurable co-processor, tightly coupled with the core General Purpose Processor (GPP). This, allows for a fast integration in existing software at a 
small cost in terms of additional area. This polymorphic architecture uses the FPGAs embedded PowerPC running at $300 \mathrm{MHz}$ as a core GPP, with a main data memory running at $100 \mathrm{MHz}$. The implementation is identical to the one described in [12].

\section{Performance Analysis and Related Work}

Even though the SHA-2 cores have been developed with a VIRTEX II Pro FPGA (XC2P30-7) as the target technology, they have also been implemented on a VIRTEX (XCV400-6) and a VIRTEX II (XC2V2000-6), in order to compare with the related art.

SHA 256 hash function core: The proposed SHA256 hash function core has been compared with the most recent and most efficient related art, for both the cores proposed in the academia and the best commercial core currently available,as far as it is known by the authors. The obtained comparison figures are presented in Table 1 . When compared with the most recent academic work [14, $8]$ the results show higher throughputs, from $17 \%$ up to $98 \%$, while achieving a reduction in area above $25 \%$ and up to $42 \%$. These results suggest a significant improvement to the Throughput per Slice (TP/Slice) metric in the range of $100 \%$ to $170 \%$. When compared with the commercial SHA256 core from Helion [15], the proposed core suggests an identical area value (less $7 \%$ ) while achieving a $40 \%$ gain to the throughput, resulting in an improvement of $53 \%$ to the Throughput per Slice metric. Note that from the analyzed cores, ours is

Table 1. SHA256 core performance comparison

\begin{tabular}{|l||c|c||c|c||c|c|}
\hline Architecture & Sklav[14] & Our & McEv.[8] & Our & Helion[15] & Our \\
\hline \hline Device & XCV & XCV & XC2V & XC2V & XC2PV-7 & XC2PV-7 \\
\hline IV & cst & yes & cst & yes & cst & yes \\
\hline Slices & 1060 & 764 & 1373 & 797 & 815 & 755 \\
\hline BRAMS & $\geq 1$ & 1 & $\geq 1$ & 1 & 1 & 1 \\
\hline Freq. (MHz) & 83 & 82 & 133 & 150 & 126 & 174 \\
\hline Cycles & n.a. & 65 & 68 & 65 & n.a. & 65 \\
\hline ThrPut (Mbit/s) & 326 & 646 & 1009 & 1184 & 977 & 1370 \\
\hline TP/Slice & $\mathbf{0 . 3 1}$ & $\mathbf{0 . 8 4}$ & $\mathbf{0 . 7 4}$ & $\mathbf{1 . 4 9}$ & $\mathbf{1 . 2}$ & $\mathbf{1 . 8 3}$ \\
\hline
\end{tabular}

the only one capable of loading the Initialization Vector $(I V)$. In the proposed FPGA implementation the logic required for the $I V$ loading is located between registers as depicted in Figure 3. If the $I V$ loading mechanism were not present the reconfigurable logic located in the CLB of the final register would be unused. Thus one can say that the $I V$ loading mechanism is implemented at zero cost. Since this loading is performed with only an additional multiplexer located between registers, it does not influence the critical path of the circuit, as confirmed by the implementation results. The structure proposed by McEvoy [8] also has 
message padding hardware. This message padding is performed once to the end of the message, and has no significant cost when implemented in software. Thus the majority of the proposed cores and commercial core do not include the hardware for this operation. McEvoy does not give figures for the individual cost of this extra hardware. All the SHA256 cores have the data expansion hardware.

SHA 512 hash function core: Table 2 presents the implementation results for our core and the most significant related art. The figures presented also suggest a significant reduction to the required reconfigurable area, from $25 \%$ up to $60 \%$, while achieving a speedup to the hashing function. When compared with [14], the core that requires less area from those compared, the proposed core requires $25 \%$ less reconfigurable logic while a throughput increase of $85 \%$ is achieved, resulting in a Throughput per Slice metric improvement of $165 \%$. From the known proposed SHA512 cores, the unrolled core proposed by Lien in [16] is the only one capable of achieving a higher throughput. However, this throughput is only $4 \%$ higher, while requiring twice as much area (100\% more) as the one proposed in this paper. It should also be noticed that the results presented by Lien in [16], do not include the data expansion module, that would most likely influence the final throughput rate, not to mention the required area. Even in this case the proposed core indicates a Throughput per Slice metric $77 \%$ higher. All other analyzed cores have even lower values for this efficiency metric. Table 2 also presents the values for the VIRTEX II Pro implementation, for which the core was originally developed.

Table 2. SHA512 core performance comparison

\begin{tabular}{|l||c|c|c|c||c|c||c|}
\hline Architecture & Sklav[14] & Lien [16] & Lien [16] & Our & McEv.[8] & Our & Our \\
\hline \hline Device & XCV & XCV & XCV & XCV & XC2V & XC2V & XC2VP \\
\hline Expansion & yes & no & no & yes & yes & yes & yes \\
\hline IV & cst & cst & cst & yes & cst & yes & yes \\
\hline Slices & 2237 & $2384^{1}$ & $3521^{1}$ & 1680 & 2726 & 1666 & 1667 \\
\hline BRAMS & n.a. & n.a. & n.a. & 2 & $\geq 1$ & 1 & 1 \\
\hline Freq. (MHz) & 75 & 56 & 67 & 70 & 109 & 121 & 141 \\
\hline Cycles & n.a. & n.a. & n.a. & 81 & 84 & 81 & 81 \\
\hline ThrPut (Mbit/s) & 480 & 717 & 929 & 889 & 1329 & 1534 & 1780 \\
\hline TP/Slice & $\mathbf{0 . 2 1}$ & $\mathbf{0 . 3}^{1}$ & $\mathbf{0 . 2 6}^{1}$ & $\mathbf{0 . 5 3}$ & $\mathbf{0 . 4 9}$ & $\mathbf{0 . 9 2}$ & $\mathbf{1 . 0 1}$ \\
\hline
\end{tabular}

Polymorphic implementation of the SHA-2 cores: In order to integrate the proposed core in the existing software applications and to easily test the cores, they were integrated into the MOLEN polymorphic processor [13]. In this processor the cores are integrated has a CCU, that can directly access the main memory and communicates with the GPP via a set of exchange registers. The core is evoked as the equivalent software function call. In order to use the proposed cores as CCU units for the MOLEN processor, some additional logic

${ }^{1}$ These values do not include the expansion data block, that in our architecture has a cost of 224 slices. 
is required. The CCU for the SHA256 core requires 994 Slices using in total $7 \%$ of the available resources of the XC2VP30 FPGA. The CCU for the SHA512 core requires 1806 Slices using in total $13 \%$ of the available resources. In this functional test, the CCU is running with same clock frequency as the main data memory, operating at $100 \mathrm{MHz}$. Table 3 presents the speedup achieved with the use of this hardware core, when compared with the pure software algorithm. The values presented are for the SHA256 kernel function. The values suggest a

Table 3. SHA256 polymorphic performances

\begin{tabular}{|c||c|c||c|c|c|}
\hline \multicolumn{1}{|c||}{} & \multicolumn{2}{c||}{ Hardware } & \multicolumn{2}{c|}{ Software } & \\
\hline \multirow{2}{*}{ Bits } & Cycles & $\begin{array}{c}(\mathrm{Mbps}) \\
\text { ThrPut }\end{array}$ & Cycles & $\begin{array}{l}\text { (Mbps) } \\
\text { ThrPut }\end{array}$ & $\begin{array}{c}\text { Kernel } \\
\text { SpeedUp }\end{array}$ \\
\hline \hline 512 & 354 & 434 & 30402 & 5.05 & 85 \\
\hline 1024 & 552 & 556 & 60546 & 5.07 & 109 \\
\hline $128 \mathrm{k}$ & 50088 & 785 & 7718646 & 5.09 & 153 \\
\hline
\end{tabular}

speedup up to 153 times for the SHA256 hash function, which is achieved when the total size of the data is sufficiently large to compensate the initialization of the core, achieving a throughput of $785 \mathrm{Mbit} / \mathrm{s}$. When only one data block is hashed the initialization time is still relevant, reducing the speedup to 85 times. When at least two data block are sent, the initialization becomes less significant, allowing already a speedup of $109 \%$. The SHA512 CCU is capable of achieving a maximum throughput of $1.2 \mathrm{Gbit} / \mathrm{s}$.

\section{Conclusions}

The proposed hardware rescheduling and reutilization schemes for the SHA-2 algorithm implementations, allow for an improvement of both performance and area resources. With the operation rescheduling, we were able to reduce the critical path in a similar manner as in the loop unrolling, without duplicating the required hardware neither using more complex data expansion schemes. This rescheduling also allows the usage of a well balanced pipeline structure that does not need additional control logic, and where both stages are always being used. The required reconfigurable resources are also significantly reduced due to the way the Digest Message is added to the intermediate values, requiring less multiplexers and adders. By adding and loading the variables $A$ and $E$ through the round hardware, area can also be saved and one less computational cycle is required to add the Digest Message. Experimental results shown a significant gain compared to the existing commercial cores and related academia art. For the SHA256 hash function, the proposed core is capable of achieving a $17 \%$ higher throughput with an area reduction of $42 \%$. When compared with the Helion commercial core a $40 \%$ higher throughput is achieved while reducing the required area by $7 \%$. As an efficiency measure, the Throughput per Slice metric 
has been improved by $53 \%$ for the considered commercial core and more than $100 \%$ when compared with the related academic art. The SHA512 hash function implementation suggest identical results, requiring $25 \%$ less reconfigurable resources than the smallest related art while achieving a $85 \%$ higher throughput. Even when compared with the unrolled architectures, the proposed core is capable of achieving identical throughputs, only $4 \%$ slower than the fastest proposal, which uses loop unrolling, for a 50\% area reduction. These values indicate an improvement to the Throughput per Slice metric of at least $77 \%$ and up to $165 \%$. On a VIRTEX II Pro FPGA, the proposed cores are capable of a throughput of $1.37 \mathrm{Gbit} / \mathrm{s}$ for the SHA256 and $1.78 \mathrm{Gbit} / \mathrm{s}$ for the SHA512, with only 755 and 1667 slices usage, respectively.

\section{References}

1. Dadda, L., Macchetti, M., Owen, J.: The Design of a High Speed ASIC Unit for the Hash Function SHA-256 (384, 512). In: DATE, IEEE Computer Society (2004) $70-75$

2. Macchetti, M., Dadda, L.: Quasi-pipelined hash circuits. In: IEEE Symposium on Computer Arithmetic, IEEE Computer Society (2005) 222-229

3. Dadda, L., Macchetti, M., Owen, J.: An ASIC design for a high speed implementation of the hash function SHA-256 (384, 512). In Garrett, D., Lach, J., Zukowski, C.A., eds.: ACM Great Lakes Symposium on VLSI, ACM (2004) 421-425

4. Grembowski, T., Lien, R., Gaj, K., Nguyen, N., Bellows, P., Flidr, J., Lehman, T., Schott, B.: Comparative analysis of the hardware implementations of hash functions SHA-1 and SHA-512. In Chan, A.H., Gligor, V.D., eds.: ISC. Volume 2433 of Lecture Notes in Computer Science., Springer (2002) 75-89

5. McLoone, M., McCanny, J.V.: Efficient single-chip implementation of SHA-384 \& SHA-512. proc. of IEEE International Conference on Field-Programmable Technology (2002) 311-314

6. Sklavos, N., Koufopavlou, O.: Implementation of the SHA-2 hash family standard using FPGAs. The Journal of Supercomputing 31 (2005) 227248

7. Ting, K.K., Yuen, S.C.L., Lee, K.H., Leong, P.H.W.: An FPGA Based SHA-256 Processor. In Glesner, M., Zipf, P., Renovell, M., eds.: FPL. Volume 2438 of Lecture Notes in Computer Science., Springer (2002) 577-585

8. McEvoy, R.P., Crowe, F.M., Murphy, C.C., Marnane, W.P.: Optimisation of the SHA-2 family of hash functions on FPGAs. IEEE Computer Society Annual Symposium on Emerging VLSI Technologies and Architectures (ISVLSI'06) (2006) 317322

9. Michail, H.E., Kakarountas, A.P., Selimis, G.N., Goutis, C.E.: Optimizing SHA-1 hash function for high throughput with a partial unrolling study. In Paliouras, V., Vounckx, J., Verkest, D., eds.: PATMOS. Volume 3728 of Lecture Notes in Computer Science., Springer (2005) 591-600

10. NIST: Announcing the standard for secure hash standard, FIPS 180-1. Technical report, National Institute of Standards and Technology (1995)

11. NIST: The keyed-hash message authentication code (HMAC), FIPS 198. Technical report, National Institute of Standards and Technology (2002)

12. (Omitted due to the blind review submission) 
13. Vassiliadis, S., Wong, S., Gaydadjiev, G.N., Bertels, K., Kuzmanov, G., Panainte, E.M.: The Molen polymorphic processor. IEEE Transactions on Computers (2004) $1363-1375$

14. Sklavos, N., Koufopavlou, O.: On the hardware implementation of the SHA-2 $(256,384,512)$ hash functions. proc. of IEEE International symposium on Circuits and systems (ISCAS 2003) (2003) 25-28

15. HELION: Fast SHA-2 (256) hash core for xilinx FPGA. http://www.heliontech. com/ (2005)

16. Lien, R., Grembowski, T., Gaj, K.: A 1 Gbit/s partially unrolled architecture of hash functions SHA-1 and SHA-512. In: CT-RSA. (2004) 324-338

\section{Appendix I - SHA-2 Operations}

In this appendix the several operations for the SHA2 algorithm are described. In Table 4 the logical operations $C h, M a j, \Sigma_{i}$, and $\sigma_{i}$ are presented, where $\oplus$ represents the bitwise $X O R$ operation, $\wedge$ the bitwise $A N D$ operation, $\operatorname{ROT} R^{n}(x)$ the right rotation operation by $n$ bits, and $S H R^{n}(x)$ the right shift operation by $n$ bits.

Table 4. SHA256 and SHA512 functions

\begin{tabular}{|r|c|}
\hline Designation & Function \\
\hline \hline Maj $(\mathrm{x}, \mathrm{y}, \mathrm{z})$ & $(x \wedge y) \oplus(x \wedge z) \oplus(y \wedge z)$ \\
\hline $\mathrm{Ch}(\mathrm{x}, \mathrm{y}, \mathrm{z})$ & $(x \wedge y) \oplus(\bar{x} \wedge z)$ \\
\hline$\sum_{0}^{\{256\}}(x)$ & $\operatorname{ROTR}^{2}(x) \oplus \operatorname{ROTR}^{13}(x) \oplus \operatorname{ROTR}^{22}(x)$ \\
\hline$\sum_{1}^{\{256\}}(x)$ & $\operatorname{ROTR}^{14}(x) \oplus \operatorname{ROTR}^{18}(x) \oplus \operatorname{ROTR}^{41}(x)$ \\
\hline$\sigma_{0}^{\{256\}}(x)$ & $\operatorname{ROTR}^{7}(x) \oplus \operatorname{ROTR}^{18}(x) \oplus \operatorname{SHR}^{3}(x)$ \\
\hline$\sigma_{1}^{\{256\}}(x)$ & $\operatorname{ROTR}^{17}(x) \oplus \operatorname{ROTR}^{19}(x) \oplus \operatorname{SHR}^{10}(x)$ \\
\hline$\sum_{0}^{\{512\}}(x)$ & $\operatorname{ROTR}^{28}(x) \oplus \operatorname{ROTR}^{34}(x) \oplus \operatorname{ROTR}^{39}(x)$ \\
\hline$\sum_{1}^{\{512\}}(x)$ & $\operatorname{ROTR}^{14}(x) \oplus \operatorname{ROTR}^{18}(x) \oplus \operatorname{ROTR}^{41}(x)$ \\
\hline$\sigma_{0}^{\{512\}}(x)$ & $\operatorname{ROTR}^{1}(x) \oplus \operatorname{ROTR}^{8}(x) \oplus \operatorname{SHR}^{7}(x)$ \\
\hline$\sigma_{1}^{\{512\}}(x)$ & $\operatorname{ROTR}^{19}(x) \oplus \operatorname{ROTR}^{61}(x) \oplus \operatorname{SHR}^{6}(x)$ \\
\hline
\end{tabular}

